\title{
THA and TKA in patients with cardiovascular disease: What makes the risk of perioperative bleeding?
}

\author{
Dzupa $\mathrm{V}^{1}$, Motovska $\mathrm{Z}^{2}$, Widimsky $\mathrm{P}^{2}$, Waldauf $\mathrm{P}^{3}$, Ondrakova $\mathrm{M}^{2}$, Bartoska $\mathrm{R}^{1}$, Jezek $\mathrm{M}^{1}$, \\ Lena $\mathrm{T}^{1}$, Popelka $\mathrm{O}^{1}$, Krbec $\mathrm{M}^{1}$
}

Department of Orthopaedics and Traumatology, Third Faculty of Medicine of Charles University and University Hospital Kralovske Vinohrady, Prague, Czech Republic. valer.dzupa@fnkv.cz

\begin{abstract}
BACKROUND: The purpose of the study was to ascertain the incidence of bleeding and ischaemic complications in patients with cardiac disease after total hip arthroplasty (THA) and total knee arthroplasty (TKA).

METHODS: In total, 477 patients (289 women, 188 men) with known history of cardiac disease or thromboembolic disease treated with surgery in 2010-2013, were enrolled in the study. Perioperative prevention of thromboembolic disease using low-molecular-weight heparins was applied in all the patients. The data that could have an impact on the development of monitored perioperative complications, were observed.

RESULTS: Complications occurred in $55(11.6 \%)$ patients: bleeding complications in 32, ischaemic in 19, and both in four patients. Complications were found in $13(12.0 \%)$ patients after THA and in $6(9.5 \%)$ patients after TKA. Bleeding complications were observed in 17 patients after THA and TKA, ischaemic in one, and both simultaneously in one patient.

Bleeding complications occurred insignificantly more frequently after THA and TKA $(p=0.094)$; however, this difference was statistically significant after adjustment for risk factors $(p=0.003)$. On the contrary, ischaemic complications were significantly more frequent after other skeletal surgeries $(p=0.014)$. Nevertheless, this difference was not statistically significant after the adjustment $(p=0.880)$. The comparison of the risk of complications in patients after THA with that in patients after TKA showed no significant difference $(p=0.580)$.

CONCLUSION: The study showed a significantly higher incidence of bleeding complications in patients after THA and TKA compared to other surgeries of the musculoskeletal system in patients with a history of cardiac disease. Bleeding complications cannot be detected in advance (Tab. 1, Ref. 16). Text in PDF www.elis.sk. KEY WORDS: perioperative bleeding, ischaemic complications, total hip replacement, total knee replacement.
\end{abstract}

\section{Introduction}

Bleeding and ischaemic complications belong to the most serious perioperative problems after skeletal surgeries. These complications may have an important impact on the result of surgery and quality of life (1-7). The development of such complications is influenced by a number of known factors such as age, weight, history of cardiac or vascular diseases, antithrombotic medication in the preoperative period, hypercoagulable state $(1,5,8,9)$.

${ }^{1}$ Department of Orthopaedics and Traumatology, Third Faculty of Medicine of Charles University and University Hospital Kralovske Vinohrady, Prague, Czech Republic, ${ }^{2}$ Third Department of Internal Medicine - Cardiology, Third Faculty of Medicine of Charles University and University Hospital Kralovske Vinohrady, Prague, Czech Republic, and ${ }^{3}$ Department of Anaesthesiology and Resuscitation, Third Faculty of Medicine of Charles University and University Hospital Kralovske Vinohrady, Prague, Czech Republic

Address for correspondence: V. Dzupa, Department of Orthopaedics and Traumatology, Third Faculty of Medicine of Charles University and University Hospital Kralovske Vinohrady, Srobarova 50, CZ-100 34 Prague, Czech Republic.

Phone: +420.267.162431, Fax: +420.267.163173

Acknowledgements: The study was supported by a research grant of the Czech Ministry of Health IGA no. NT 11506-6/2010.
Total hip arthroplasty (THA) and total knee arthroplasty (TKA) implantations rank among surgeries that are most burdened by perioperative and postoperative blood losses. The purpose of this study was to find out whether the risk of bleeding and ischaemic complications after THA and TKA in patients with cardiac disease is higher than that after other orthopaedic and traumatological surgeries of the musculoskeletal system.

\section{Material and methods}

The study group included 477 patients undergoing surgery due to an orthopaedic disease or injury to the musculoskeletal system. In their history, the patients had any of diseases as follows: ischaemic heart disease, atrial fibrillation, stroke, deep vein thrombosis in lower limbs, pulmonary embolism, or heart valve surgery. Our study was a subanalysis of the PRAGUE 14 Study (registered on www.ClinicalTrials.gov under the identifier NCT01897220) with the total number of 1200 enrolled patients followed in 2011-2013, undergoing any neurosurgery, or thoracic, abdominal, urological or orthopaedic surgery (10).

The following data were registered in the database system eCRF (web medium APEX, Oracle Application Express 10g, data exported via RapidMiner 5.3): data related to cardiovascular his- 
tory (myocardial infarction, percutaneous coronary intervention, coronary artery bypass graft, atrial fibrillation, valvular disease, prosthetic valve, stroke, deep vein thrombosis, pulmonary embolism) and the history of antithrombotic medication, data related to the surgery and perioperative period (basic haematological and biochemical test results, anticoagulation perioperative prophylaxis, duration of surgery, perioperative complications), intraoperative bleeding complications (requiring prolongation of surgery) and postoperative bleeding complications (requiring surgical revision or transfusion without revision), postoperative ischaemic complications (acute stroke, myocardial infarction, first or recurrent heart failure, pulmonary embolism, deep vein thrombosis, acute limb ischaemia) and the number of deaths.

Basic data (age, sex, height, weight, surgery type, medication) were evaluated using descriptive statistical methods. The hypotheses were tested by the regression analysis method both without and with adjustment for demonstrated risk factors (sex, age, height, weight, smoking, hypertension, chronic heart failure, percutaneous coronary intervention, warfarin versus warfarin discontinuation three or more days before the surgery, ASA score, general vs local anaesthesia, acute vs elective procedure, haemoglobin < $100 \mathrm{~g} / \mathrm{L}$, thrombocytes $>300.10^{12} / \mathrm{L}$, thrombocytes $<150.10^{12} / \mathrm{L}$, creatinine $>100 \mathrm{mmol} / \mathrm{L}$ ); statistical software Stata 13 was used.

\section{Results}

The study group of 477 patients comprised 289 women (61 \%) and 188 men (39\%); of whom 272 (57\%) underwent acute surgery, and 205 (43\%) elective surgery. THA was performed in 108 (23\%) patients and TKA in 63 (13\%) patients. An overview of the surgeries per anatomical site is presented in Table 1.

The age of all patients ranged from 33 to 99 years (mean age 76 years; median age 78 years), while in patients after THA and TKA, the age ranged from 46 to 94 years (mean age 73 years; median age 75 years).

Complications were observed in 55 (11.6\%) patients: bleeding complications in 32 (6.9\%) and ischaemic complications in 19(4.0\%) patients, while both types of complications were seen at the same time in 4 (0.8\%) patients. Complications were observed in 13 (12.0\%) patients after THA and 6 (9.5 \%) patients after TKA. Bleeding complications were experienced by 17 patients after THA and TKA, ischaemic by one patient, and a combination of bleeding and ischaemia was seen in one patient.

Tab. 1. Surgeries performed in patients of the study group per anatomical site.

\begin{tabular}{lcc}
\hline & Number & Percentage \\
\hline Upper limb & 53 & $11.1 \%$ \\
\hline Lower limb & 393 & $82.4 \%$ \\
-THA & 108 & $22.6 \%$ \\
-TKA & 63 & $13.2 \%$ \\
-another & 222 & $46.6 \%$ \\
\hline Spine & 25 & $5.2 \%$ \\
\hline Pelvis & 6 & $1.3 \%$ \\
\hline In total & 477 & $100 \%$ \\
\hline
\end{tabular}

No statistical difference was found upon comparison of all complications in patients after THA or TKA and other operated patients ( $p=0.911$; odds ratio $0.75 ; \mathrm{CI}=0.5 ; 1.6$ ); insignificant difference persisted also after adjustment for demonstrated risk factors (age, general anaesthesia) ( $\mathrm{p}=0.260$; odds ratio 1.47; $\mathrm{CI}=0.75$; 2.85).

Bleeding complications were seen insignificantly more frequently after THA and TKA ( $\mathrm{p}=0.094$; odds ratio 1.78; $\mathrm{CI}=0.91$; 3.49); however, after adjustment (significant: general anaesthesia, borderline insignificant: sex, hypertension, warfarin administration), this difference was statistically significant ( $\mathrm{p}=0.003$; odds ratio 3.2 ; $\mathrm{CI}=1.49$; 7.0 ).

On the contrary, ischaemic complications occurred significantly more frequently after other skeletal surgeries $(\mathrm{p}=0.014$; odds ratio 6.22; $\mathrm{CI}=1.44$; 26.88); however, after adjustment (significant: age; borderline insignificant: thrombocytes $<150.10^{12} / \mathrm{L}$, acute surgery), this difference was statistically insignificant ( $\mathrm{p}=$ 0.880 ; odds ratio 1.14 ; $\mathrm{CI}=0,2 ; 3,71$ ).

After comparison of the risk of complications in patients after THA with that in patients after TKA we did not observe any significant difference ( $\mathrm{p}=0.580$; odds ratio $1.34 ; \mathrm{CI}=0.49 ; 3.71$ ), and after adjustment (significant: age, general anaesthesia; borderline insignificant: warfarin administration, smoking, thrombocytes > $\left.300.10^{12} / \mathrm{L}\right)$ this difference remained insignificant $(\mathrm{p}=0.481$; odds ratio 1.52; $\mathrm{CI}=0.47 ; 4.9)$. The difference between bleeding complications in patients after THA compared to TKA was also insignificant ( $\mathrm{p}=0.375$; odds ratio $2.0 ; \mathrm{CI}=0.55 ; 4.81$ ), and after adjustment (significant: age, general anaesthesia, thrombocytes $>300.10^{12} / \mathrm{L}$, borderline insignificant: warfarin administration, smoking) this difference remained insignificant $(\mathrm{p}=0.273$; odds ratio 2.0; $\mathrm{CI}=0.57$; 7.13). Finally, not even the difference in ischaemic complications between patients after THA compared to those after TKA was significant ( $p=0.715$; odds ratio $0.59 ; \mathrm{CI}=0.04$; 9.67); adjustment was not possible due to the low incidence of ischaemic complications.

\section{Discussion}

In our study, which was focused on the risk of bleeding and ischaemic complications in patients after acute and elective surgeries of the musculoskeletal system, we noted a significantly higher risk of bleeding complications after elective procedures, and contrariwise, a significantly higher risk of ischaemic complications after acute procedures (11). The higher risk of ischaemic complications in patients after acute procedures was closely related to higher age of patients. We wanted to find out whether the increased risk of bleeding complications in elective procedures could be affected by the most commonly scheduled procedures, i.e. THA and THK. Patients after arthroplasty implantation were slightly younger in our group, but we believe that this minor difference could not have had any impact on the risk.

As shown by our study, bleeding complications occurred significantly more frequently in patients after THA and TKA. We believe this to be especially due to the fact that considerable resection of connective tissue and significant exposure of spongy bone occur after both procedures, meaning that perioperative and subsequent postoperative blood losses are significant in both THA 
and TKA. Moreover, perioperative anticoagulation prophylaxis, which is recommended in all these patients, is consistently applied in our patients using low-molecular-weight heparins $(2,12)$.

Other procedures performed in patients of the study group included also surgeries where higher perioperative blood losses are seen (scoliosis, arthroplasty of the shoulder, internal fixation of the pelvic fracture); however, the number of these procedures was considerably lower in our group compared to procedures associated with minimum perioperative bleeding (cerclage of the olecranon, open reduction and internal fixation for forearm fractures, proximal femoral nail and dynamic hip screw for hip fractures, cerclage of the patella, non-reamed tibial nail for tibial fractures, open reduction and internal fixation for ankle fractures).

Ischaemic complications are considered serious for the patient's prognosis as well as for the long-term result of surgery. We did not demonstrate an increased incidence of such complications in our group in patients after THA or TKA.

Numerous studies reported high postoperative blood losses after TKA implantation $(6,13,14)$. As shown by our study, the difference in the incidence of bleeding complications in patients after THA compared to that after TKA was not significant. In our opinion, this is a very important finding especially for patients with the history of cardiac disease who require perioperative anticoagulation prophylaxis for both types of arthroplasty implantations (2). Higher perioperative blood losses in THA need not mean a higher incidence of subsequent bleeding complications.

Given the significantly higher incidence of bleeding complications and insignificant difference in the incidence of ischaemic complications in patients after THA or TKA compared to patients after other orthopaedic procedures, we can ask whether consistent perioperative anticoagulation prophylaxis in patients with arthroplasty may be the reason for the reduced incidence of ischaemic complications or the cause of increased incidence of bleeding complications (4, 5, 7, 12, 15, 16). We cannot answer this speculative question based on the results achieved in our study.

\section{Conclusion}

The study showed a significantly higher incidence of bleeding complications in patients after THA and TKA compared with other orthopaedic and traumatological surgeries of the musculoskeletal system in patients with a history of cardiac disease. The incidence of ischaemic complications in patients after THA or TKA was insignificantly lower than in those after other musculoskeletal surgeries. The difference in the incidence of bleeding and ischaemic complications in patients after THA compared to TKA was not significant.

\section{References}

1. Bushnell BD, Horton JK, McDonald MF, Robertson PG. Perioperative medical comorbidities in the orthopaedic patient. J Am Acad Orthop Surg 2008; 16: 216-227.

2. Douketis JD, Spyropoulos AC, Spencer FA, Mayr M, Jaffer AK, Eckman MH, Dunn AS, Kunz R. Perioperative management of antithrombotic therapy antithrombotic therapy and prevention of thrombosis, 9th ed: American College of Chest Physicians Evidence-Based Clinical Practice Guidelines. Chest 2012; 141 (Suppl): e326S-350S.

3. Geerts WH, Pineo GF, Heit JA, Bergqvist D, Lassen MR, Colwell CW, Ray JG. Prevention of venous thromboembolism. The Seventh ACCP Conference on Antithrombotic and Thrombolytic Therapy. Chest 2004; 126: 338S-400S.

4. Lee HL, Chiu KY, Yiu KH, Ng FY, Yan CH, Chan PK. Perioperative antithrombotic management in joint replacement surgeries. Hong Kong Med J 2013; 19: 531-538.

5. Oberweis BS, Nukala S, Rosenberg A, Guo Y, Stuchin S, Radford MJ, Berger JS. Thrombotic and bleeding comlications after orthopedic surgery. Am Hearth J 2013; 165: 427-433.

6. Petrlicek J, Stehlik J, Sadovsky P, Musil D, Mezera V. The effect of tranexamic acid on blood loss after primary unilateral total knee arthroplasty: prospective single-centre study. Acta Chir Orthop Traumatol Cech 2015; 82: 418-423.

7. Vetter TR, Boudreaux AM, Papapietro SE, Smith PW, Taylor BB, Porterfield JR Jr. The perioperative management of patients with coronary artery stents: surveying the clinical stakeholders and arriving at a consensus regarding optimal care. Am J Surg 2012; 204: 453-461.

8. Cluett J, Caplan J, Yu W. Preoperative cardiac evaluation of patients with acute hip fracture. Am J Orthop 2008; 37: 32-36.

9. Motovska Z. Management of antiplatelet therapy in patients at risk for coronary Stent Thrombosis undergoing non-cardiac surgery. Drugs 2011; 71: 1797-1806.

10. Widimsky P, Motovska Z, Havluj L, Ondrakova M, Bartoska R, Bittner L, Dusek L, Dzupa V, Knot J, Krbec M, Mencl L, Pachl J, Grill R, Haninec P, Waldauf P, Gürlich R. Perioperative cardiovascular complications versus perioperative bleeding in consecutive patients with known cardiac disease undergoing non-cardiac surgery. Focus on antithrombotic medication. The PRAGUE-14 registry. Neth Heart J 2014; 22: 372-379.

11. Dzupa V, Waldauf P, Motovska Z, Widimsky P, Ondrakova M, Bartoska R, Jezek M, Lena T, Popelka O, Krbec M. Risk comparison of bleeding and ischemic perioperative complications after acute and elective orthopaedic surgery in patients with cardiovascular disease. Arch Orthop Trauma Surg 2016; 136: 907-911.

12. Leijtens B, Kremers van de Hei K, Jansen J, Koëter S. High complication rate after total knee and hip replacement due to perioperative bridging of anticoagulant therapy based on the 2012 ACCP guideline. Arch Orthop Trauma Surg 2014; 134: 1335-1341.

13. Polednik Z, Pastucha M. [The use of a pneumatic turniquet in primary total knee replacement surgery: are lower blood losses while?] (in Czech) Ortopedie 2014; 8: 137-140.

14. Frisch NB, Wessell NM, Charters MA, Yu S, Jeffries JJ, Silverton CD. Predictors and complications of blood transfusion in total hip and knee arthroplasty. J Arthroplasty 2014; 29 (Suppl 9): 189-192.

15. Biondi-Zoccai G, Lotrionte M, Agostoni P, Abbate A, Fusaro M, Burzotta F, Testa L, Sheiban I, Sangiorgi G. A systematic review and meta-analysis on the hazards of discontinuing or not adhering to aspirin among 50279 patients at risk for coronary artery disease. Eur Heart J 2006; 27: 2667-2674.

16. Decker RC, Foley JR, Moore TJ. Perioperative management of the patient with cardiac disease. J Am Acad Orthop Surg 2010; 18: 267-277.

Received June 26, 2016. Accepted July 18, 2016. 